\title{
Eckel, Jan/Schild, Georg (Hrsg.): 1968. Verdichtung des Wandels und globaler Moment (Tübinger Vorlesungen), 220 S., Mohr Siebeck, Tübingen 2019. Kraushaar, Wolfgang: Die 68er-Bewegung International. Eine illustrierte Chronik 1960-1969, 4 Bde., 2004 S., Klett-Cotta, Stuttgart 2018.
}

\section{Werner Bührer}

Angenommen: 5. Januar 2021 / Online publiziert: 21. Januar 2021

(C) Der/die Autor(en) 2021, korrigierte Publikation 2021

Die Unterschiede zwischen den beiden Publikationen könnten in Anlage und Format größer kaum sein: auf der einen Seite ein schmaler, 220-seitiger Band mit essayistischen Studien auf der Basis der einschlägigen Literatur, auf der anderen ein vierbändiges, insgesamt knapp über 2.000 Seiten umfassendes, chronologisch angelegtes Panoptikum der ,Sixties“, zusammengesetzt aus Zeitungsartikeln, Briefen, Erinnerungen, Abbildungen von Plakaten und Buchcovern sowie Fotos - ein wahrer Koloss. Damit hätten sich damals, in ausreichender Zahl eingesetzt, ohne Weiteres Barrikaden bauen lassen. Doch trotz dieser Unterschiede verbinden beide Werke zahlreiche Gemeinsamkeiten. Sie zeigen die globale Dimension von ,1968', die Vielschichtigkeit und Komplexität der Ereignisse, die ,Jugendlichkeit ‘ der weltweiten Rebellion, die entscheidende Rolle kultureller Manifestationen sowie soziokultureller Milieus - und eben die „Verdichtung des Wandels“, wie es im Untertitel des Essaybandes heißt, um das Jahr 1968: „Daraus floss ein gemeinsamer Grundstrom politischer Energien, so unterschiedlich sich die konkreten Forderungen auch ausnahmen“ (Eckel/Schild, S. 35).

Der Essayband ist aus einer Vorlesungsreihe an der Universität Tübingen hervorgegangen; dort lehren oder lehrten alle sieben Beiträger, fünf sind Historiker unterschiedlicher Fachrichtung. Ihr Anliegen ist es, ,1968‘ in ,größere historische Entwicklungslinien der zweiten Jahrhunderthälfte" einzuordnen, und zwar anhand einer „räumlich weitgespannten Auswahl an symptomatischen Einzelstudien“ (S. 4), die sich von Prag und Tübingen bis Mexiko City und San Francisco erstreckt. Mehr oder weniger explizit weisen alle den von einigen, Veteranen ' erhobenen heroisierenden Anspruch zurück, die 68er-Bewegung habe ,ex nihilo eine neue, bessere Gesellschaft geschaffen“, so die Herausgeber (S. 2), oder, so Eckel in seinem Aufsatz über 1968 in der Bundesrepublik, es habe sich um einen „,nachgeholten Gründungsakt“

W. Bührer $(\bowtie)$

Technische Universität München, München, Deutschland

E-Mail: Werner.Buehrer@tum.de 
gehandelt, „durch den der nur äußerlich freiheitliche Rahmen der Bundesrepublik endlich mit innerem demokratischen Leben gefüllt“ (S. 13) und der ,restaurative Muff des Bonner Biedermeier erstmals kulturell durchlüftet worden wäre" (S. 14).

Dagegen betont Eckel die Widersprüchlichkeit des Aufbruchs, den er in der kulturellen Sphäre beispielsweise an der Gleichzeitigkeit des Erfolgs der progressiven Düsseldorfer Künstlerkneipe und Konzertbühne „Creamcheese“ und Heintjes Single „Mama“, des Fortbestehens des „Kuppeleiparagraphen“ und des Entstehens des weltweit „wohl größten“ (S. 13) Marktes für Pornografie festmacht. Die von den 68ern verstärkten Tendenzen zur politischen Liberalisierung und die „Lebensstilrevolutionen“ stellt er in den Kontext breiterer Prozesse des Wandels: „die Ankunft eines Massenwohlstands, die gewachsene institutionelle Stabilität [...] und nicht zuletzt eine immense Verjüngung der Gesellschaft“. Mit anderen Worten: der „Glaube an traditionelle Hierarchien, überkommene Rollenmuster, illiberale Normierungen und vermeintliche Werte wie Ordnung, Gehorsam oder Pflichterfüllung" sei schon vor dem Ende der 1960er Jahre erodiert (S. 16). Einen weiteren Beleg für die Widersprüchlichkeit sieht Eckel in den fantasievollen, humoristischen, ironisch entlarvenden Ausdrucksformen des Protests und dem Anstieg der ,terroristischen Gewalt", die ,aus der Mitte von ,1968““ hervorgegangen sei (S. 23). Er deutet die 68erBewegung demnach ,nicht als Vorhut der gesellschaftlichen Öffnung [...], sondern als deren Produkt“, was nicht impliziere, ,ihr die historische Bedeutung abzusprechen" (S. 17). Ob er mit dieser Interpretation der aktiven, vorantreibenden Rolle der Bewegung tatsächlich gerecht wird, erscheint jedoch zweifelhaft.

Die lange Vorgeschichte unterstreichen auch Georg Schild für die USA, Johannes Großmann für Frankreich und Klaus Gestwa für die Tschechoslowakei und den Ostblock. Anders als in der Bundesrepublik kam im ,Pariser Mai“ und im ,Prager Frühling ' ein immerhin kurzfristiges Bündnis zwischen Studenten- und Arbeiterschaft zustande. Allerdings weist Gestwa zu Recht darauf hin, dass - ,ungeachtet einiger Gemeinsamkeiten“ der Geschehnisse in Ost und West - „die Unterschiede gravierender" gewesen seien (S. 103). Bernd-Stefan Grewe untersucht im Anschluss die Zusammenhänge zwischen der brutalen Niederschlagung einer friedlichen Demonstration durch das Militär und den zehn Tage später beginnenden Olympischen Spielen in Mexiko. Eine lokal- und eine popgeschichtliche Studie beschließen den Band. Bernd Jürgen Warneken, der sich als ,Tübinger 68er“ (S. 159) outet, führt den Nachweis, dass die kleine, eher beschaulich-provinzielle Universitätsstadt ,durchaus ein Subzentrum des gesellschaftlichen Aufbruchs“ war (S. 160), während Horst Tonn den weltweiten Erfolg des Musicals „Hair“ vor allem damit erklärt, dass es „die Ambivalenzen von Utopie und Endzeit, von Entfremdung und Suche nach Resonanz-Erfahrungen“ aufmerksam wahrgenommen und ,dafür eindringliche Bilder und Töne" (S. 210) gefunden habe.

Anders als die Autoren des Essaybandes, welche die Vielschichtigkeit und Komplexität der Ereignisse in den 1960er Jahren exemplarisch zu verdeutlichen versuchen, demonstriert Wolfgang Kraushaar diese durch die schiere, überwältigende Zahl chronologisch angeordneter Einträge unterschiedlicher Länge. Kraushaar, Zeitzeuge und Historiker der 1968-Bewegung in einer Person - als letzterer zweifelsohne einer der produktivsten und prominentesten - ist ein erfahrener Chronist. Davon zeugen seine 1996 erschienene vierbändige Protestchronik der Jahre 1949 bis 1959 
und seine den Zeitraum 1946 bis 1995 umfassende dreibändige Chronik „Frankfurter Schule und Studentenbewegung“ (1998). Wer letzteres Werk rezipiert hat, wird manche der Einträge der neuen Bände wiedererkennen.

Die Bebilderung ist dieses Mal noch opulenter ausgefallen. Schwarz-Weiß-Fotos dominieren eindeutig, einige Farbaufnahmen sind auch darunter. Die insgesamt etwa 1.000 Fotos haben illustrierenden Charakter, der Bildnachweis am Ende jedes Bands beschränkt sich auf den Fundort. Nur wenige Aufnahmen sind so vorbildlich ,erklärt' wie jenes aus dem Vietnamkrieg, bei dem das Datum, die Namen einer der abgebildeten Personen und des Fotografen sowie der Publikationsort erwähnt werden (Bd. 1, S. 458). Unter dem Gesichtspunkt der Quellenkritik ist der Verzicht auf eine präzise Erklärung und Kontextualisierung problematisch, doch dieses Manko erschien dem Chronisten offensichtlich nicht so wichtig. Manche Fotos laden ob ihres zunächst verblüffenden Motivs zum Nachschlagen auf, beispielsweise das, auf dem die Schauspieler Sidney Poitier und Charlton Heston sowie der Sänger Harry Belafonte anlässlich des legendären „Marsches auf Washington“ am 28. August 1963 zu sehen sind. Heston, der damals mit der Bürgerrechtsbewegung sympathisiert hatte, repräsentierte nämlich von 1998 bis 2003 als Präsident die erzkonservative National Rifle Association. Die Einträge bedienen sich meist einer neutralen, rapportierenden Sprache, mitunter enthalten sie aber auch Erklärungen oder rückblickende Einordnungen, etwa den Text zu einem Vortrag des amerikanischen Literaturwissenschaftlers Leslie A. Fiedler vom 29. Juni 1968 in Freiburg über „Postmoderne“ (Bd. 3, S. 346ff.).

Die vier Bände bilden unterschiedlich lange Zeiträume ab: der Vorgeschichte, also den Jahren 1960 bis 1966, widmet Kraushaar einen Band, den restlichen drei Jahren jeweils einen - wobei der letzte Band einen Ausblick auf das Jahr 1970 gibt und zwei Einträge zum 3. Januar 1980 enthält, dem Todestag sowohl Rudi Dutschkes als auch des Sohns von Axel Springer. Diese Gewichtung entspricht Kraushaars Deutung, nach der sich die in der ,Vorzeit“ entfaltenden Prozesse am Ende des Jahrzehnts ,,auf eine ebenso außergewöhnliche wie unvorhersehbare Weise verdichteten“ (Bd. 1, S. VIII). Der Eintrag vom 1. Januar 1960 gilt den antisemitischen Schmierereien zunächst in Köln, der vorletzte vom 31. Dezember 1969 der endgültigen Schließung des Hamburger „Star-Clubs“, in dem unter anderem die Beatles aufgetreten waren. Vier zentrale Komplexe - der 2. Juni 1967, das Attentat auf Dutschke, der ,Pariser Mai“ und die Niederschlagung des ,Prager Frühlings', außerdem die „Rote-Punkt-Aktion“ in Hannover - werden besonders minutiös dokumentiert. Immer wieder finden sich darüber hinaus farblich hervorgehobene Tafeln zu einzelnen wichtigen Organisationen (z. B. African National Congress, Black Panthers), Ereignissen (z.B. Algerienkrieg, „Große Proletarische Kulturrevolution“), oder Personen (z.B. Bertrand Russel, Herbert Marcuse, Fritz Bauer, Bobby Seale). Übrigens tauchen auch einige Einträge zu Tübingen auf und bekräftigen somit Warnekens These vom „Subzentrum des Aufbruchs“. Einmal betreibt der Chronist mit der Coverabbildung eines von ihm verfassten Buchs sogar Eigenwerbung (Bd. 4, S. 382: „Die Bombe im Jüdischen Gemeindehaus“, erschienen 2005).

Während Kraushaar die Bundesrepublik vor allem wegen der exponierten Lage im Kalten Krieg und der nachwirkenden NS-Vergangenheit als „Sonderfall“ (B. 1, S. XIV) begreift, stellen die USA für ihn das „Schlüsselland für die gesamte De- 
kade" dar (ebd., S. XVI). Entsprechend prominent thematisiert er innen- und auBenpolitische Geschehnisse wie die Bürgerrechtsbewegung und selbstverständlich den Vietnamkrieg. In den USA entstand ,mit Sit-ins, Go-ins und Teach-ins ein ganz eigener Formenkatalog, der in unzähligen Ländern adaptiert und in der Verschmelzung von Handlungs- und Öffentlichkeitselementen zu einem neuartigen Modus des Protests gemacht wurde“ (ebd., S. XVII). Deutlich mehr Aufmerksamkeit als in seinen früheren Arbeiten erfährt die kulturelle Sphäre: Theater, Literatur, Popmusik, die Festivals von Monterey und Woodstock, Drogenkonsum, Demonstrationen als „,kollektive Rauscherfahrung“, ,sexuelle Revolution“, Medien - alle wichtigen Phänomene der ,Gegenkultur" werden angemessen gewürdigt, desgleichen in der politischen Sphäre der langsame Übergang von der ,begrenzten Regelverletzung“ zum „Gewaltakt als Zauberelixier“ (ebd., S. XXVI). Bedenkenswert erscheint Kraushaars Hinweis auf die Rolle von Außenseitern wie Tariq Ali oder Daniel Cohn-Bendit als „Sprecher der Revolte“ (ebd., S. XXIVf.).

Beide Werke bereichern die Literatur zu ,1968` auf unterschiedliche Weise: Der Essayband durch die konzentrierte Erfassung der Verdichtung der lokalen und globalen Wandlungsprozesse ,um 1968', die Chronik durch die kaum zu überbietende Demonstration und Dokumentation der Vielfalt und Internationalität des Geschehens. Für das Studium der vier Bände der Chronik müssen Leserinnen und Leser reichlich Zeit mitbringen. Aber diese Investition lohnt sich unbedingt.

Funding Open Access funding enabled and organized by Projekt DEAL.

Open Access Dieser Artikel wird unter der Creative Commons Namensnennung 4.0 International Lizenz veröffentlicht, welche die Nutzung, Vervielfältigung, Bearbeitung, Verbreitung und Wiedergabe in jeglichem Medium und Format erlaubt, sofern Sie den/die ursprünglichen Autor(en) und die Quelle ordnungsgemäß nennen, einen Link zur Creative Commons Lizenz beifügen und angeben, ob Änderungen vorgenommen wurden.

Die in diesem Artikel enthaltenen Bilder und sonstiges Drittmaterial unterliegen ebenfalls der genannten Creative Commons Lizenz, sofern sich aus der Abbildungslegende nichts anderes ergibt. Sofern das betreffende Material nicht unter der genannten Creative Commons Lizenz steht und die betreffende Handlung nicht nach gesetzlichen Vorschriften erlaubt ist, ist für die oben aufgeführten Weiterverwendungen des Materials die Einwilligung des jeweiligen Rechteinhabers einzuholen.

Weitere Details zur Lizenz entnehmen Sie bitte der Lizenzinformation auf http://creativecommons.org/ licenses/by/4.0/deed.de. 